INPLASY

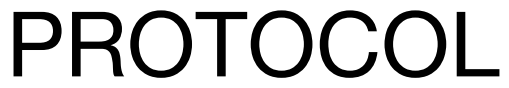

To cite: Jiang et al. Influencing factors related to internet addiction among Chinese College Students: a protocol for systematic review and meta-analysis. Inplasy protocol 2020110143. doi:

10.37766/inplasy2020.11.0143

Received: 29 November 2020

Published: 30 November 2020

Corresponding author: Jing Gao

gaojing@cdutcm.edu.cn

Author Affiliation:

Chengdu University of TCM.

Support: None.

Review Stage at time of this submission: Preliminary

searches.

Conflicts of interest:

None.

\section{Influencing factors related to internet addiction among Chinese College Students: a protocol for systematic review and meta-analysis}

Jiang, XL1; Gao, J2; Zheng, YP3.

Review question / Objective: Through meta-analysis, systematically evaluate the related influencing factors of internet addiction among Chinese College Students, and provide a scientific basis for formulating their related prevention and control measures.

Condition being studied: In China, with the popularization of the Internet and the increasing number of Internet users, Internet addiction has become increasingly prominent as a social problem. Among the people who use the Internet, college students have the largest proportion. Internet addiction affected by many factors such as family, school and sociodemography, and there are many studies have conducted relevant discussions, but the conclusions are not nearly the same, so our meta-analysis aims to comprehensively investigate the influencing factors associated with internet addiction among Chinese College Students. and to provide a scientific basis for formulating their related prevention and control measures.

INPLASY registration number: This protocol was registered with the International Platform of Registered Systematic Review and Meta-Analysis Protocols (INPLASY) on 30 November 2020 and was last updated on 30 November 2020 (registration number INPLASY2020110143).

\section{INTRODUCTION}

Review question / Objective: Through meta-analysis, systematically evaluate the related influencing factors of internet addiction among Chinese College Students, and provide a scientific basis for formulating their related prevention and control measures.
Condition being studied: In China, with the popularization of the Internet and the increasing number of Internet users, Internet addiction has become increasingly prominent as a social problem. Among the people who use the Internet, college students have the largest proportion. Internet addiction affected by many factors 
such as family, school and sociodemography, and there are many studies have conducted relevant discussions, but the conclusions are not nearly the same, so our meta-analysis aims to comprehensively investigate the influencing factors associated with internet addiction among Chinese College Students. and to provide a scientific basis for formulating their related prevention and control measures.

\section{METHODS}

Search strategy: Articles related to Internet addiction among Chinese College Students between 2010 and 2020 will be retrieved from the Chinese periodical data-bases of Chinese Biomedical Literature Database (CBM), China National Knowledge Infrastructure (CNKI), VIP Database, WanFang Database and from PubMed, the Cochrane Library, Web of Science, Embase. We search the following keywords: "Internet addition", "college students/ university students/Medical Students", "risk factors/ influencing factors / population at risk" and "China/Chinese". Languages will be restricted to English and Chinese. In addition, relevant articles will be manually searched.

Participant or population: The Chinese College Students with internet addiction (as diagnosed using Young's Internet Addiction Diagnostic Questionnaire (YDQ) or the Chen Internet Addiction Scale (CIAS)).

Intervention: Risk factors of internet addiction among Chinese College Students.

Comparator: The Chinese College Students without internet addiction.

Study designs to be included: case-control studies, cohort studies, and crosssectional surveys.

Eligibility criteria: Inclusion criteria included: (1) Studies published from January 2010 to December 2020; (2) Research types include case-control studies, cohort studies, and crosssectional surveys; (3) the research objects are full-time Chinese college students or vocational college students who are 18-25 years old; (4) At least one risk factor has been reported. Exclusion criteria: (1) articles unrelated to the purpose of the study; (2) valid data cannot be extracted from the study; (3) data is incomplete or repeated publication.

Information sources: Articles related to Internet addiction among Chinese College Students between 2010 and 2020 will be retrieved from the Chinese periodical databases of Chinese Biomedical Literature Database (CBM), China National Knowledge Infrastructure (CNKI), VIP Database, WanFang Database and from PubMed, the Cochrane Library, Web of Science, Embase.

Main outcome(s): The influencing factors related to internet addiction among Chinese College Students such as family, school and sociodemography.

Quality assessment / Risk of bias analysis: According to selection criteria, data extraction will be completed independently by two researchers. Disagreements will be solved by discussion or a third reviewer. For missing information, we will contact the correspondent authors for completed data. The following information will extract from the literature: first author, year of publication, investigation time and area, sampling method, sample size, gender composition, and the scale used for Internet addition, influencing factors. Evaluation tools recommended by the wellestablished Newcastle-Ottawa scale (NOS) based on three categories, and a total score of at least 6 was considered high quality.

Strategy of data synthesis: Stata $\mathbf{1 1 . 0}$ software will be used for the analysis. The odds ratio (OR), standard mean difference (SMD) and 95\% confidence interval (Cl) used as the effect indexes for the counting data and measurement data, respectively. The heterogeneity among the included results will be analyzed using $Q$ test, and 
the heterogeneity will quantitatively determined by combining with 12 . When there is significant statistical heterogeneity among the studies $(P<0.1,12>50 \%)$, the combined effect size of the random effect model was adopted, Otherwise, the fixed effect model be adopted.

Subgroup analysis: If there is heterogeneity, we will conduct a subgroup analysis, we will take an analysis of subgroup according to characteristic of participants, such as race, study location, majors and gender.

Sensibility analysis: The sensitivity analyses will be conducted by changing the statistical analysis model.

Language: English.

Country(ies) involved: China.

Keywords: China; College students; Internet addiction; Meta-analysis; Influencing factors.

Contributions of each author:

Author 1 - Xiaolin Jiang - Contributed to search databases, screen articles and draft the manuscript.

Author 2 - Jing Gao - Contributed to search related studies and analysis statistics.

Author 3 - Yuping Zheng - Contributed to literature retrieval and risk assessment of bias. 